\title{
Near-field scanning optical microscopy of photonic crystal nanocavities
}

\author{
Koichi Okamoto, ${ }^{a}$ Marko Lončar, Tomoyuki Yoshie, and Axel Scherer \\ Department of Electrical Engineering, California Institute of Technology, Pasadena, California 91125-9300 \\ Yueming Qiu and Pawan Gogna \\ In Situ Technology and Experiments System Section, Jet Propulsion Laboratory, California Institute of \\ Technology, MS 302-306, 4800 Oak Grove Drive, Pasadena, California 91109
}

(Received 23 September 2002; accepted 20 January 2003)

\begin{abstract}
Near-field scanning optical microscopy was used to observe high-resolution images of confined modes and photonic bands of planar photonic crystal (PPC) nanocavities fabricated in active InGaAsP material. We have observed the smallest optical cavity modes, which are intentionally produced by fractional edge dislocation high- $Q$ cavity designs. The size of the detected mode was roughly four by three lattice spacings. We have also observed extended dielectric-band modes of the bulk PPC surrounding the nanocavity by geometrically altering the bands in emission range and eliminating localized modes out of the emission range. (C) 2003 American Institute of Physics. [DOI: $10.1063 / 1.1559646]$
\end{abstract}

Photonic crystals, ${ }^{1}$ and planar photonic crystals (PPC) in particular, have recently attracted attention as a promising platform for realization of compact and efficient nanocavities $^{2,3}$ and lasers. $^{4-8}$ In most of these reports, microphotoluminescence was used to characterize the structures. On the other hand, near-field scanning optical microscopy (NSOM) has recently been used as a powerful alternative method to analyze local electromagnetic field distributions in fabricated nanophotonic structures. ${ }^{9-17}$ Gérard et al. ${ }^{14}$ reported NSOM measurements of active PPC with spectral emission in the infrared region and Shin et al. ${ }^{15}$ reported the near-field investigation of the lasing modes in PPC lasers. However, in both studies, uncoated optical fibers were used and, therefore, it was not possible to obtain high spatially resolved near-field images of the field distribution inside the cavity. Also, both studies analyzed large hexagonal cavities (empty lattice cavities), which support many modes with rather large mode volumes. In this letter, we report the results of NSOM of very small PPC cavities based on fractional edge dislocations. ${ }^{2,3,8}$ The metal-coated fiber tip enables us to distinguish between localized cavity modes and propagating far-field modes, and to obtain more precise mode profiles when the tip probes into holes of PPCs. The best resolution in our system is as small as $50 \mathrm{~nm}$.

The experimental setup for the NSOM measurement is shown in Fig. 1. We used a twin-SNOM system manufactured by OMICRON, capable of both illumination mode (Imode) and the collection mode (C-mode) measurements. For the I mode, continuous-wave light from a He-Ne laser (633 $\mathrm{nm}$ ) was used to pump the structures through the optical fiber tip. The photoluminescence (PL) signal was detected through the reflective objective lens. The excitation power of the $\mathrm{He}-\mathrm{Ne}$ laser, before coupling into the optical fiber, was 1 $\mathrm{mW}$. For the $\mathrm{C}$ mode, a $780 \mathrm{~nm}$ diode laser, operated with 20 ns long pulses of $2 \mu$ s periodicity, was focused on the sample through the refractive objective lens and the optical fiber tip was used to detect the PL signal. The excitation pump beam

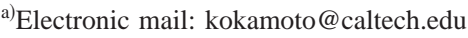

spot was several tens of $\mu \mathrm{m}^{2}$. In both modes, the PL signals were distinguished from the reflected light of the excitation laser by using the colored glass filter with a cutoff wavelength of $850 \mathrm{~nm}$, and detected with a high-sensitivity (fW) InGaAs photodetector. The optical fiber tip was metal coated and the aperture size at the end of the tip was $150 \mathrm{~nm}$. The fiber tip is positioned at the dither piezodevice and shearforce detector in order to control the distance between the tip and the sample surface $(\approx 10 \mathrm{~nm})$ and to obtain a topographic image of the sample.

The PPC nanocavities described in this work are very similar to those used to realize low-threshold lasers described in our previous publication. ${ }^{8}$ The most important difference from the cavities analyzed in Ref. 8 is the omission of central defect hole, and therefore $Q$ factors are limited to about 1000 , according to our theoretical predictions. Optical emission in our structures was obtained from four $9 \mathrm{~nm}$ thick InGaAsP quantum well $(\mathrm{QW})$ layers $(\mathrm{Eg}=1.55 \mu \mathrm{m})$ separated by $20 \mathrm{~nm}$ thick InGaAsP barrier layers $(\mathrm{Eg}=1.22 \mu \mathrm{m})$, and placed in the center of a $330 \mathrm{~nm}$ thick InGaAsP slab, grown on the top of InP substrate. The emission from QWs was found to be in the range of 1300 to $1650 \mathrm{~nm}$. The PPC structure is a free-standing membrane patterned with triangular lattice of holes within which cavity based on fractional edge dislocation is defined. Details of the fabrication procedures are presented in Ref. 8.

Figure 2(a) shows the topographic image of the entire structure obtained by the shear-force microscopy. The PPC structure in the center of the membrane as well as the unpatterned edges of the membrane can be seen. In Fig. 2(b), we show the near-field optical image of the same sample obtained using NSOM I mode. A bright region corresponds to the light localized in the Fabry-Perot (FP) resonator formed between the edge of the membrane and the edge of the PPC region in Fig. 2(b). We have confirmed this by conducting micro-PL measurements on this $\approx 2.1 \mu \mathrm{m}$ long resonator, and FP resonances were detected in the spectrum when the structure was pumped close to the edge [Fig. 2(c)]. 


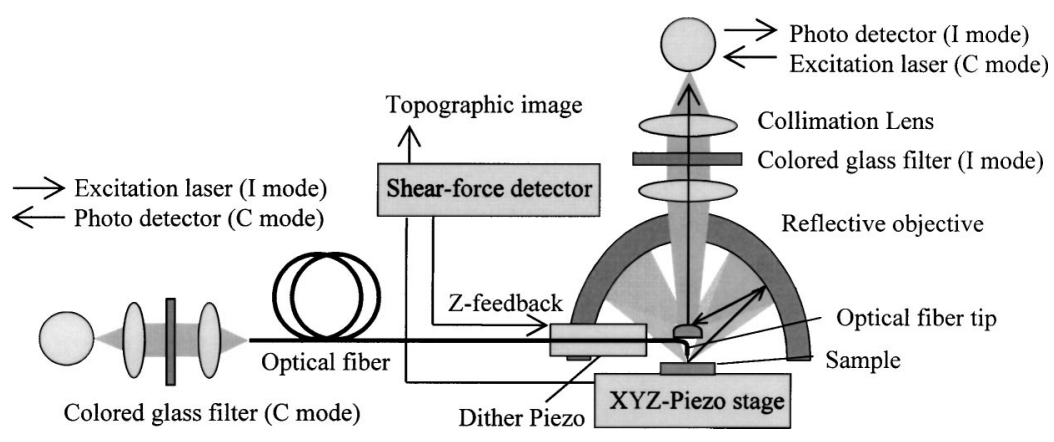

FIG. 1. Experimental setup for the NSOM measurement with illumination mode and collection mode.

Close inspection of Fig. 2(b) also reveals presence of the light localized at the center of the PPC structure. In order to investigate the origin of this signal, we have increased the spatial resolution of our NSOM and analyzed only the central region of the structure, where a nanocavity based on fractional edge dislocations exists. In Fig. 3(a), we show the scanning electron microscope (SEM) image of this central region of the device shown in Fig. 2. The periodicity of the lattice is $a=420 \mathrm{~nm}$, radius of holes is $r=135 \mathrm{~nm}$, and thickness of the slab is $d=330 \mathrm{~nm}$. This PPC geometry, with $r / a=0.32$ and $d / a=0.79$, has a band gap in the frequency range approximately $a / \lambda \in(0.25,0.33)$, that is in the wavelength range $\lambda \in(1270 \mathrm{~nm}, 1680 \mathrm{~nm})$. The elongation in this cavity was $p / a=15 \% .^{2,8}$ The cavity based on fractional edge dislocations supports two prominent resonances. These resonances correspond to doubly degenerate dipole modes of the simple single defect cavity, ${ }^{4}$ and the introduced asymmetry due to the dislocation lifts the degeneracy. The two dipole modes are linearly but orthogonally polarized, and the mode positioned at a longer wavelength can have very high $Q$. Figure 3(b) shows the results of micro-PL analysis of this structure. Two peaks positioned around $\lambda=1450 \mathrm{~nm}$ correspond to the localized dipole modes, whereas peaks above $\lambda=1600 \mathrm{~nm}$ correspond to dielectric band modes.

Figures 3(c) and 3(d) show an enlargement of the central region from Figs. 2(a) and 2(b), respectively. The bright spot seen in NSOM-PL image [Fig. 3(d)] is located at the center of the PPC structure, matching the position of the defect cavity, as shown in topographic image [Fig. 3(c)]. We have attributed this optical signal to two dipole eigenmodes of our cavity. The size of the bright spot is roughly $4.4 a$ by $3 a$. This small spot size is an indication of a small mode volume, as expected from the localized cavity modes. The NSOM images should be a superposition of two orthogonal dipole modes. These NSOM-PL results are obtained by using I mode and, therefore, the size of the bright spot is expected to contain information on the diffusion properties of free carri-
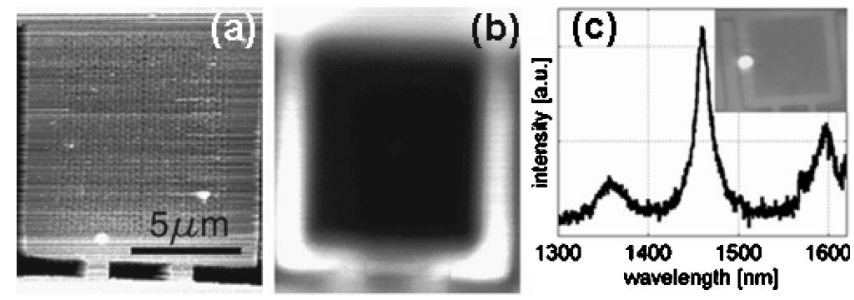

FIG. 2. (a) Topographic image of the whole structure by the shear-force microscopy. (b) Near-field PL image. (c) FP resonances detected using microphotoluminescence when structure was pumped close to the edge. Inset

shows pump spot on the structure.
Downloaded 18 Dec 2005 to 131.215 .240 .9 . Redistribution subject to AIP license or copyright, see http://apl.aip.org/apl/copyred without and (f) with a colored glass filter. ers excited by the pump beam, in addition to the information on the optical mode size. Therefore, we believe that by using I mode, we actually overestimate the size of the optical mode due to the free-carrier diffusion. However, the small size of the detected light signal is a clear indication of presence of well-confined modes in the center of our cavity. In Figs. 3(e) and 3(f), we show NSOM images without and with a cutoff colored glass filter, respectively, this time for a cavity with elongation $p / a=20 \%$. When the filter was not used [Fig. $3(\mathrm{e})]$, an interference pattern was observed. We have attributed this to the reflection of the pump $\mathrm{He}-\mathrm{Ne}$ laser light from the sample surface. On the other hand, when a filter was used, a very different result was obtained and, clearly, localized defect modes could be observed. Figure 4 shows the polarization dependence of the optical modes detected in cavity with $p / a=25 \%$, obtained using both micro-PL and NSOM approaches. As predicted by theory, two dipole modes are linearly polarized, with orthogonal polarizations. The intensities of the NSOM images with $0^{\circ}, 60^{\circ}$, and $90^{\circ}$ $\left( \pm 15^{\circ}\right)$ polarizers are in very good agreement with spectra obtained using microphotoluminescence. Therefore, we conclude that optical modes detected with NSOM correspond to confined cavity modes.

Figure 5 shows the topographic images and the corresponding NSOM images for different PPC structures, this

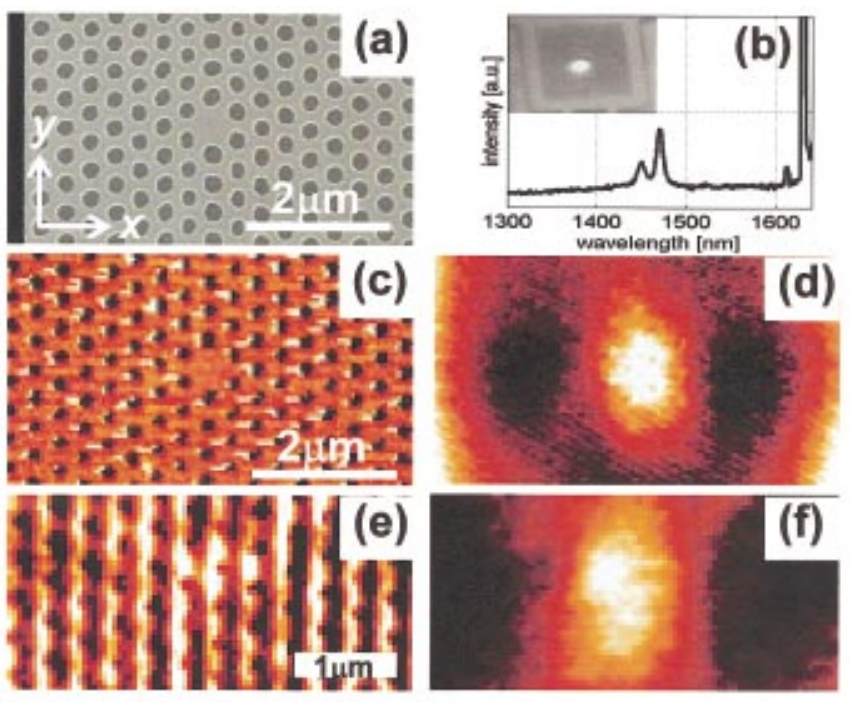

FIG. 3. (Color) (a) SEM image of the tested cavity with $p / a=15 \%$. (b) Resonances detected using micro-PL setup. Confined modes (around 1450 $\mathrm{nm}$ ) and extended dielectric band modes (above $1600 \mathrm{~nm}$ ) can be seen. (c) Topographic and (d) near-field optical image. Detected optical field corresponds to the confined cavity modes. (e) Near-field image of the cavity with

$p / a=20 \%$ obtained without and (f) with a colored glass filter.
AIP license or copyright, see http://apl.aip.org/apl/copyright.jsp 
(a)
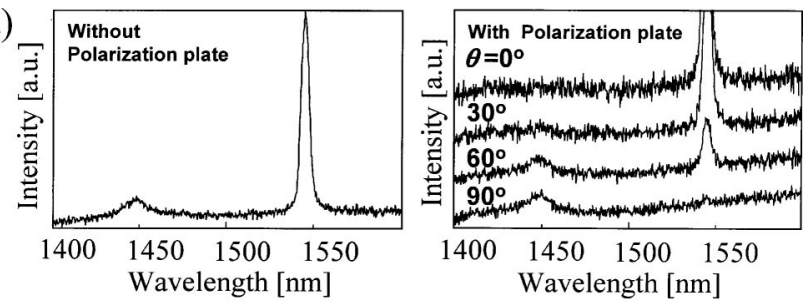

(b)

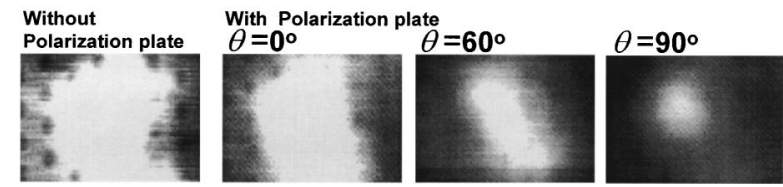

FIG. 4. (a) Micro-PL spectra and (b) near-field images taken from a sample with a parameter $p / a=25 \%$ with and without a polarization plate. A polarization parameter $\theta$ is a clockwise angle from $x$ axis defined in Fig. 3(a).

time with slightly larger holes and with the central defect hole present. Therefore, we expect dipole eigenmodes of this cavity to be moved toward shorter wavelengths. In the tested sample, they were completely pushed outside the emission range of QWs, and no localized cavity modes could be observed in the micro-PL experiment. However, we could observe several peaks that correspond to the dielectric bands of bulk PPC [Figs. 5(c) and 5(f)]. The NSOM images obtained using C-mode and shown in Figs. 5(a) and 5(b) show light localization in the dielectric region between the PPC holes. Also, positions of the air holes appear dark in this NSOM image. Therefore, we have attributed this result to the existence of the dielectric band modes in the emission region of QW material. Similar results were predicted by theory. ${ }^{10}$ Also, spectra obtained using microphotoluminescence [Fig. 5(c)] show the presence of dielectric bands at $\lambda=1555 \mathrm{~nm}$. We would like to point out that dielectric band modes, also observed in Fig. 3(b) were not detected using NSOM I mode [Figs. 3(d) and 3(f)]. We believe that it is due to localized

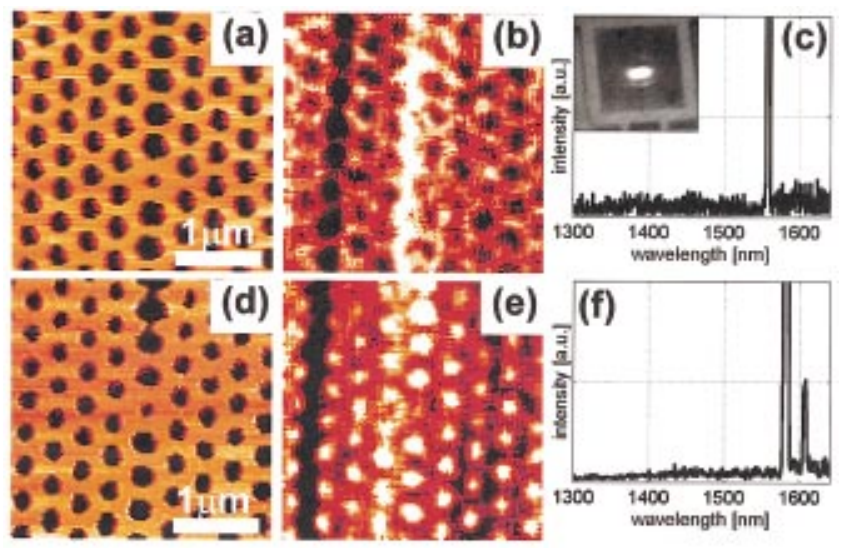

FIG. 5. (Color) (a) Topographic image and (b) near-field PL image (C mode) of PPC structure with bigger holes $(p / a=25 \%)$. (d) Topographic and (e) near-field image of another structure. (c) and (f) show spectra obtained by microphotoluminescence from structures shown in (a) and (d), respectively. pumping in the case of I mode (as opposed to the large pumping spot in $\mathrm{C}$ mode) and dielectric band modes that extend over large areas in PPC could not be excited. We have tested another geometries in PPC structures, and the NSOM results are shown in Figs. 5(d) and 5(e). Strong light intensity is observed at the positions of the air holes, this time. This phenomenon could be attributed to the presence of airband modes in the emission region of the QW material. However, we were not able to observe air-band modes in our micro-PL experiments, and only dielectric band modes were observed [Fig. 5(f)]. At present, experiments are underway to explain this phenomenon.

In conclusion, we have observed localized defect modes of the compact PPC nanocavities. In addition to localized cavity modes, we have experimentally observed dielectric band modes in bulk PPCs. We conclude that NSOM is a powerful tool for the investigation of local profiles of confined modes in nanocavities.

This work was supported by the Japan Society for the Promotion of Science (12002454), Caltech MURI Center for Quantum Networks, DARPA (MDA 972-00-1-0019), and AFOSR (F49620-01-6-0497). Two of the authors (Y.Q. and P.G.) acknowledge the partial support from the Cross Enterprise Technology Development Program at the Jet Propulsion Laboratory (under a contract with the National Aeronautics and Space Administration).

${ }^{1}$ E. Yablonvitch, Phys. Rev. Lett. 58, 2059 (1987); S. John, ibid. 58, 2486 (1987)

${ }^{2}$ J. Vučković, M. Lončar, H. Mabuchi, and A. Scherer, Phys. Rev. E 65, 016608 (2001).

${ }^{3}$ T. Yoshie, J. Vučković, A. Scherer, H. Chen, and D. Deppe, Appl. Phys. Lett. 79, 4289 (2001).

${ }^{4}$ O. Painter, R. K. Lee, A. Scherer, A. Yariv, J. D. O'Brien, P. D. Dapkus, and I. Kim, Science 284, 1819 (1999).

${ }^{5}$ H. G. Park, J. K. Hwang, J. Huh, H. Y. Ryu, and Y.-H. Lee, Appl. Phys. Lett. 79, 3032 (2001).

${ }^{6}$ H.-Y. Ryu, S.-H. Kim, H.-G. Park, J.-K. Hwang, Y.-H. Lee, and J.-S. Kim, Appl. Phys. Lett. 80, 3883 (2002).

${ }^{7}$ T. Yoshie, O. B. Shchekin, H. Chen, D. G. Deppe, and A. Scherer, Electron. Lett. 38, 967 (2002).

${ }^{8}$ M. Lončar, T. Yoshie, A. Scherer, P. Gogna, and Y. Qiu, Appl. Phys. Lett. 81, 2680 (2002).

${ }^{9}$ M. L. M. Balisteri, D. J. W. Klunder, F. C. Blom, A. Driessen, W. J. M. Hoekstra, J. P. Korterik, L. Kuipers, and N. F. van Hulst, Opt. Lett. 24, 1829 (1999)

${ }^{10}$ G. W. Bryant, E. L. Shirley, L. S. Goldner, E. B. McDaniel, J. W. P. Hsu, and R. J. Tonucci, Phys. Rev. B 58, 2131 (1998).

${ }^{11}$ S. Fan, A. Ian, and J. D. Joannopoulos, Appl. Phys. Lett. 75, 3461 (1999).

${ }^{12}$ D. Mulin, C. Girard, G. Colas des Francs, M. Spajer, and D. Courjon, J. Microsc. 202, 110 (2000).

${ }^{13}$ A. L. Campillo, J. W. P. Hsu, C. A. White, and A. Rosenberg, J. Appl. Phys. 89, 2801 (2001).

${ }^{14}$ D. Gérard, L. Berguiga, F. de Fornel, L. Salomon, C. Seassal, X. Letartre, and P. Viktorovitch, Opt. Lett. 27, 173 (2002).

${ }^{15}$ D.-J. Shin, S.-H. Kim, J.-K. Hwang, H.-Y. Ryu, H.-G. Park, D.-S. Song, and Y.-H. Lee, IEEE J. Quantum Electron. 38, 857 (2002).

${ }^{16}$ T. Baba, H. Yamada, and A. Sakai, Appl. Phys. Lett. 77, 1584 (2000).

${ }^{17}$ P. Kramper, A. Birner, M. Agio, C. M. Soukoulis, F. Müller, U. Gösele, J. Mlynek, and V. Sandoghdar, Phys. Rev. B 64, 023102 (2001). 